\title{
Making questions with tone: Polar question formation in Kinyarwanda
}

\author{
Alexander Jarnow*
}

\begin{abstract}
Kinyarwanda is a Bantu language with one phonemic $(\mathrm{H})$ tone (Kimenyi 2002). This can phonetically realized as high, low, rising, and falling. This talk addresses the tonological discrepancy between declaratives and polar questions in Kinyarwanda. Kimenyi (1980) Briefly addresses Kinyarwanda polar questions and describes them as "a rising pitch at the sentence final position". This generalization captures crucially cannot predict polar questions in which there is no LHL contour at the end of the sentence. I argue that what polar questions share is (a) suspension of downstep on the rightmost lexical $\mathrm{H}$ and (b) deletion of all word-final prosodic H. Kinyarwanda forms a prosodic structure that takes the scope of the question. This expands on Richards (2010) analysis of wh-questions. Kinyarwanda marks the right edges of prosodic words using boundary tones, similar to Chichewa (Kanerva 1990; Myers 1996).
\end{abstract}

Keywords. Kinyarwanda; tonology; Bantu; prosody; polar question

1. Introduction. The aim of this paper is to describe the tonological patterns of polar question formation in Kinyarwanda and provide a theoretical analysis that accounts for the observed facts. I will use evidence from polar question and wh-question formation to show that a phonological process simply inserting a high tone on the penultimate syllable is inadequate to account for the observed facts in Kinyarwanda. Previous works on question formation in Kinyarwanda and related Bantu languages stipulate a high tone on the penultimate syllable (Kimenyi 1980; Downing 1989). A prosodic domain between the compementizer and the rightmost lexical high tone in the utterance more adequately describes the observed tonal pattern.

The tonological analysis presented here has implications for how tone is manipulated in tonal languages to form polar questions. This work presents a detailed description of the patterns of polar question formation in various contexts and posits a tonological analysis to account for them. My analysis of Kinyarwanda also fits within a larger typological framework of polar question formation and uses specific tonological features of Kinyarwanda to account for the observed data. This work extends current understanding of prosodic phrasing in Kinyarwanda and adds a theoretical explanation to account for the formation of polar questions using only the manipulation of tone.

\section{Background.}

2.1. TonE In KinYARWANDA. Kinyarwanda is a Bantu language with one phonemic tone. Phonetically this phonemic tone leads to a contrast that is realized as high and low, but but there is evidence that high tone is the only lexical tone (Kimenyi 2002). Contrary to contour tone systems that designate tones as having distinct pitch contours such as Mandarin Chinese (Maddieson 2005), Kinyarwanda is a register tone system in which tones are not associated with an absolute frequency, but distinguished by their pitch level relative to each other (Hyman 1993).

${ }^{*}$ Thank you to Christophe Hararimana for invaluable data, Claire Halpert for advice and direction, as well as to the audience at LSA 2020 for helpful discussion. Author: Alexander Jarnow, University of Minnesota (jarno011@umn.edu). 
Rising and falling tones also surface on certain syllables, but these are the result of contrastive vowel length and the mora as the tone-bearing unit. In contexts with long vowels (two moras), a high tone can be associated with either the first or second mora (Goldsmith \& Mpiranya 2010). A high tone associated with the first mora of a long vowel surfaces as falling intonation, or HL. Similarly, a high tone associated with the second mora of a long vowel surfaces as rising intonation, or LH. This allows three types of surface tones on long vowels: rising (LH), falling (HL), or a low, level intonation (LL). These possibilities are shown in (1).
a. umw-aámi
LH (sounds like rising tone)
1-king
'king'
b. umw-áana HL (sounds like falling tone)
1-child
'child'
c. umu-untu LL (sounds like low, level tone)
1-human
'human'

(Myers 2003) demonstrates that $\mathrm{F}_{0}$ peaks are realizes either later or earlier in the syllable and provides the moraic tone assignment shown in (2).

(2) Moraic high tone assignment in Kinyarwanda (Myers 2003):
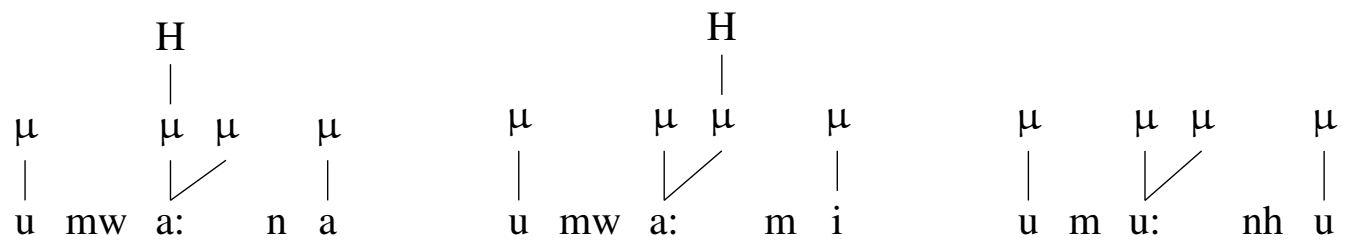

Tones in Kinyarwanda serve both lexical and grammatical functions. (3) shows two lexical minimal pairs that vary only in their tonal patterns. Adding a high tone to kuvuna changes the meaning to a different verb. This is possible of both verbs and nouns.
a. ku-vun-a
INF-rescue-ASP
'to rescue'
b. ku-vún-a
INF-break-ASP
'to break'
c. umu-ryáango
1-door
'door'
d. umu-ryaango
1-family
'family'

2.2. Tonological PROCESSES. Apart from the lexical tone inventory, moraic tonal assignment rules, and grammatical tone assignment, there are also numerous tonological processes that affect the phonetic tones that surface in words and phrases. This section will address some 
of the more common and observable tonological processes, as these will become relevant to account for how tones surface on longer phrases and utterances.

In many Bantu languages tones can be lexically assigned to a syllable, and then shift or spread to other syllables in the word or across word boundaries. In Kinyarwanda, tones only have the ability to spread to the left (referred to as tone anticipation by Kimenyi (2002)). Leftward tone spread is a relatively rare process in Bantu and rightward tone spreading is much more common (Odden \& Bickmore 2014).

One example of this leftward high tone spread is found in infinitive verbs. The infinitive marker initiates the in which the infinitive marker $k u$ - receives a high tone only if there is a lexical high tone on the first syllable of the verb stem (Kimenyi 2002):
a. gu-sinzir-a
INF-sleep-ASP
'to sleep'
b. ku-rúm-a $\rightarrow$ kú-rúm-a
INF-bite-ASP
'to bite'

In (4a), there is no high tone on the verb root and thus the infinitive marker also does not bear a high tone (there are three allophones of the infinitive morpheme: $g u$ - before voiceless consonants and $k w$ - before vowels). In (4b), the stem does have a high tone and thus high tone spreads leftward to the infinitive marker. The pitch tracks for these two words are represented in (5) and (6):

(5)

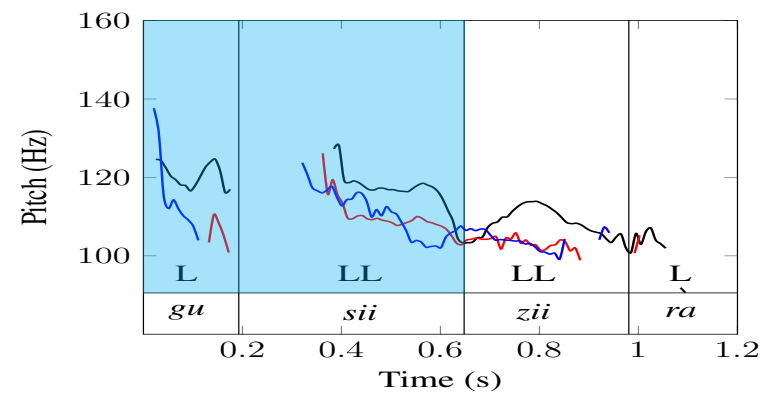

(6) kuruma - to bite

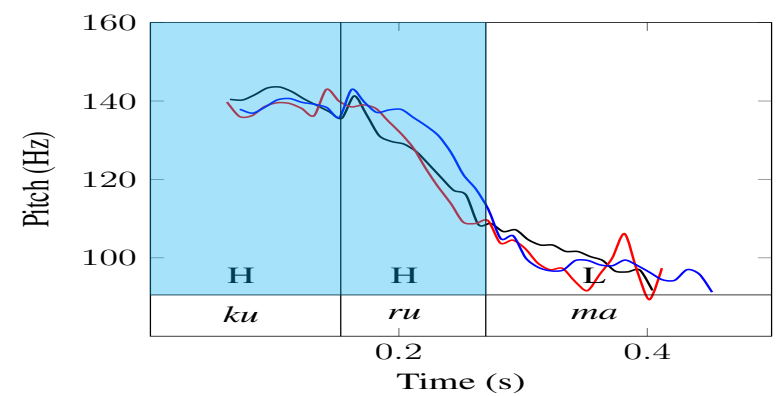

Another common tonological process attested in Bantu languages is downstep. Downstep is a phenomena in which each successive high tone is lowered and 'a new ceiling is established for subsequent high tones' (Connell 2016). Kinyarwanda exhibits downstep in nearly all utterances, and an example is shown in (7) \& (8). 
(7) umu-zíkí

1-music

'music'

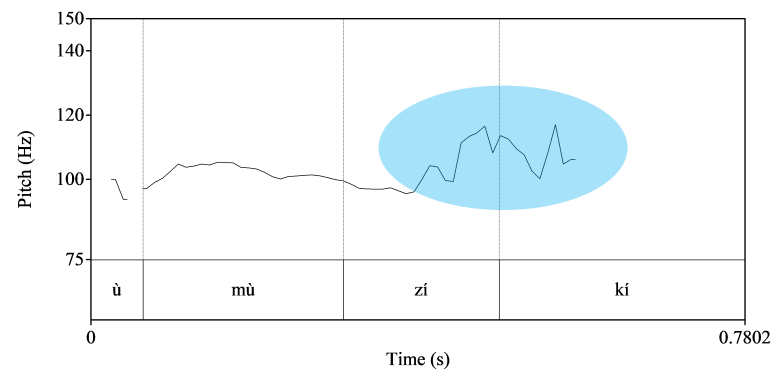

(8) n-dimó n-da-téger-á

1SG-PROG 1SG-PROG-listen-ASP

umu-zíkí

1-music

'I am listening to music.'

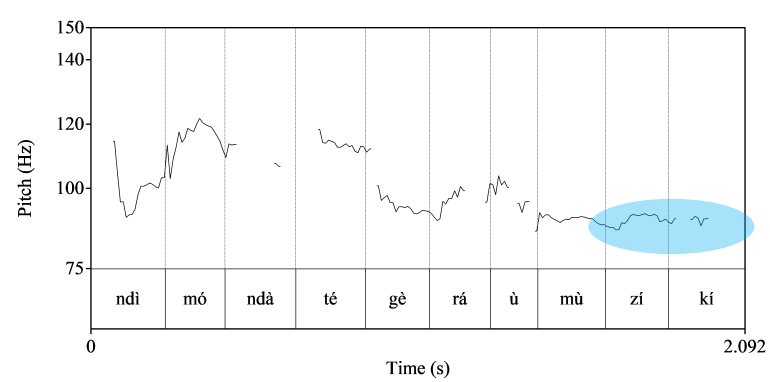

Downstep plays an important role in Bantu polar question formation as suspension of downstep is attested in numerous Bantu languages. Suspension of downstep as a polar question formation process is addressed in Section 3.

2.3. Prosodic Phrasing. Prosodic boundaries and how prosodic phrases are formed in Kinyarwanda will contribute the background for part of my central claim. Phonological rules that apply at the left or right edge of a constituent are useful diagnostics for determining the boundaries of prosodic phrases as laid out in (Selkirk 1980a,b). Tones are a very common way to mark the edges of prosodic boundaries and tend to cluster around recurring processes (Hyman 1990).

There are no lexical high tones in (9), but when this word is followed by another to make a bigger prosodic domain, a high tone surfaces on the final syllable of the word.

(9)
umw-anditsi
1-writer
'writer'

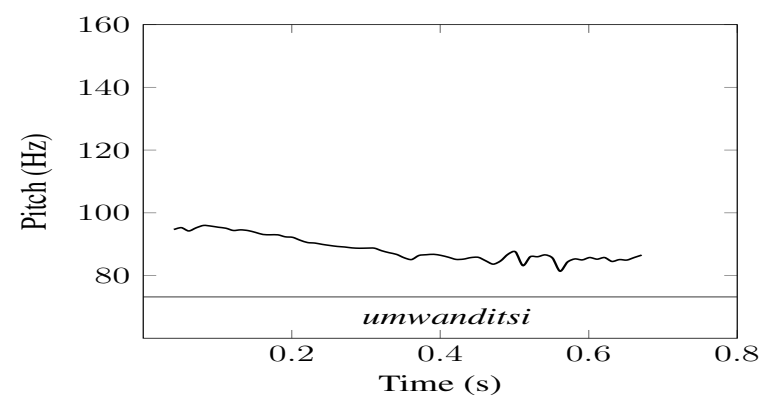


umw-anditsi w-igi-tabo

1-writer POSS-7-book

'the writer of the book'

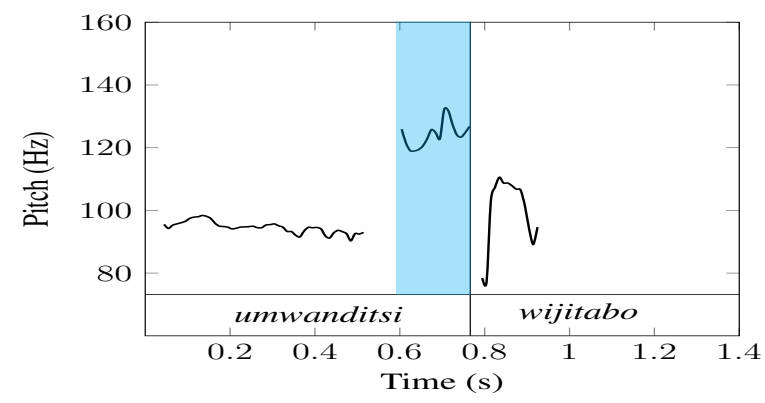

Unlike (9) in isolation, (10) contains the same word but with a different tonal contour. In (10) the word-final syllable has a high tone. Applying the parameters laid out in 2.3, a high boundary tone targets the right edge of prosodic words. Since utterance-final high tones are never observed in Kinyarwanda, I will assume that a phonological rule or higher prosodic structure deletes any high tones of prosodic words in utterance-final position.

3. Polar questions. Polar questions can be marked by different processes, including movement, an overt question particle, or by manipulating intonation and prosody. While is it common for languages to employ prosodic change in conjunction with another way of marking a polar question, languages without overt morphosyntactic differences, including movement or overt question particles, must rely solely on prosodic or tonological means to convey polar question meaning. This section will introduce comparisons between declaratives and polar questions and show how Kinyarwanda forms polar questions only by manipulating tone.

In Rialland (2007)'s robust study on question prosody in African languages, 78 Bantu languages were identified as constructing polar questions without overt interrogative morphemes. She notes that these can be broadly divided into two categories: those that use high pitch/tone as a question marker and those that don't. Other than high tone, polar questions can also be indicated by employing suspension of downstep, penultimate lengthening, register expansion, or other tonological processes that don't involve marking a question explicitly with a high tone.

In Jita, a Bantu language closely related to Kinyarwanda, Downing (1989) asserts that polar questions are formed by inserting a high tone on the first syllable and spreading that high tone rightward through the first lexical high tone in the utterance. This is seen in (11).
a. Ba:-ku-fúli-ye
they-you-wash.for-TENSE
'They have washed (something) for you.'
b. Bá:-kú-füli-ye
'Have they washed (something) for you?'

If there is not an underlying high tone in a polar question, all syllables through the penult have a raised pitch.
a. Ba:-fuli-ye
they-wash.for-TENSE
'They have washed (something) fro (someone).'
b. Bá:-fúlí-ye
'Have they washed (something) for (someone)?' 
Kinyarwanda is like many Bantu languages in that it lacks an overt question particle or a syntactic movement process to form polar questions. Thus, Kinyarwanda must make use of prosodic and tonological processes in order to differentiate between declaratives and polar questions.

My focus in this paper is the role of tone in polar question formation in Kinyarwanda. Existing descriptions (Kimenyi 1980, 2002) only briefly address polar question marking in the context of wh-questions, characterizing it as a sentence-final pitch manipulation:

Note that the yes-no question construction is marked only by intonation - consisting of rising pitch at the sentence final position - which distinguishes it from the simple declarative sentence. (Kimenyi 1980; 75)

This description is correct in that polar questions in Kinyarwanda are marked strictly prosodically (i.e. without an overt interrogative morpheme). For example, the minimal pair in (13) does generally fit this description.

$$
\begin{array}{lll}
\text { a-rimó } & \text { a-ra-jend-á } \quad \text { kwi-soko } \\
\text { 3SG-PROG } & \text { 3SG-PROG-go-ASP LOC-market }
\end{array}
$$

'S/he is going to the market.'

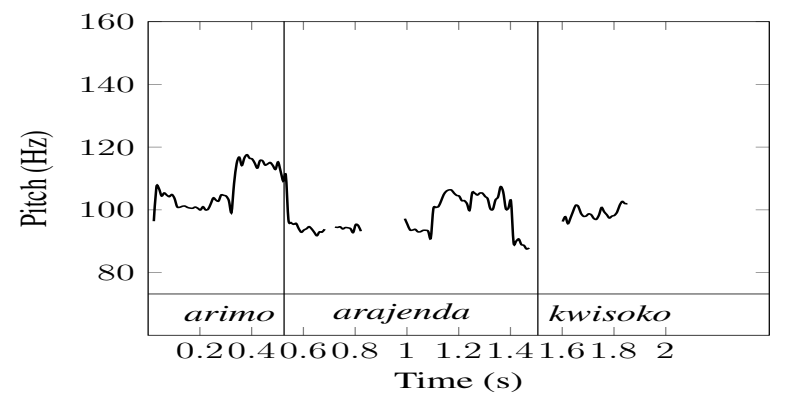

$$
\text { a-rímó a-ra-jend-a kwi-sőko }
$$

3SG-PROG 3SG-PROG-go-ASP LOC-market

'is s/he going to the market?'

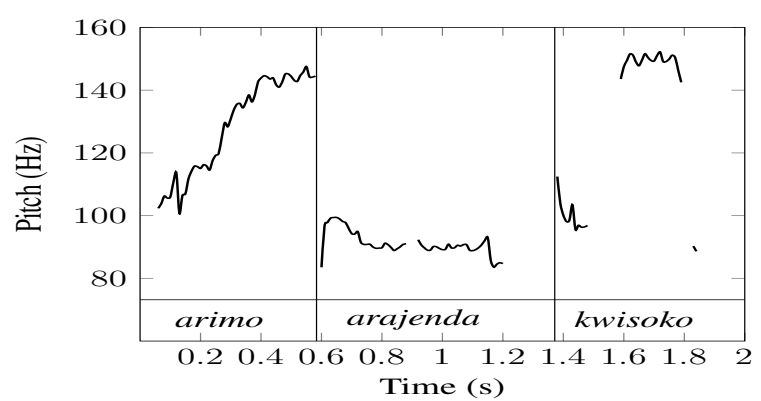

In (14), there is indeed a rising pitch in the sentence-final position. To be more precise, the rising intonation takes place in the penultimate syllable and then falls again in the utterancefinal position. This also conforms with the observation that word-final syllables have a high tone and utterance-final syllables never have a high tone. However, there is more to the picture than a simple rise in intonation in the penultimate syllable of the utterance. First, the pitch of the the auxiliary is raised. The pitch of the verb is flattened and slightly lowered from its 
declarative counterpart, although the mean pitch values remain similar. The crucial difference between (13) and (14) is the pitch in the utterance-final word, kwisoko. In the polar question a high tone exists where there is none in the declarative and this high tone is higher than other high tones in the sentence.

4. Description and analysis. Chewa and Tumbuka also display polar question formation that on the surface is similar in some ways to what is observed in Kinyarwanda. In Chewa, polar questions have an obligatory fall-rise contour over the last two syllables of the question (15a), while Tumbuka polar questions have a high pitched fall-fall contour over the last two syllables (15b) (Downing \& Mtenje 2011):

a. mu-ku-fúná khóofíí? (Downing \& Mtenje 2011)

2PL-TAM-want coffee

'Do you want coffee?'

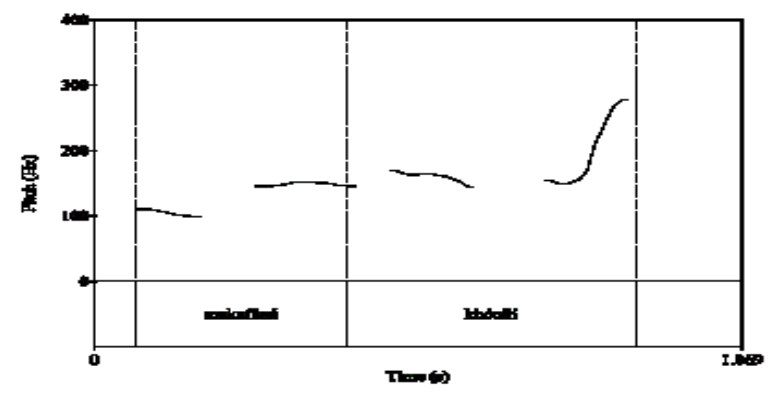

b. káasi, ni dokotala péera uyo wa-ku-vwira mu-sambíizi ku-sukúulúu?

Q COP 1.doctor only 1.REL 1 SBJ-TAM-help 1-teacher LOC-school

'Does only the doctor help the teacher at the school?'

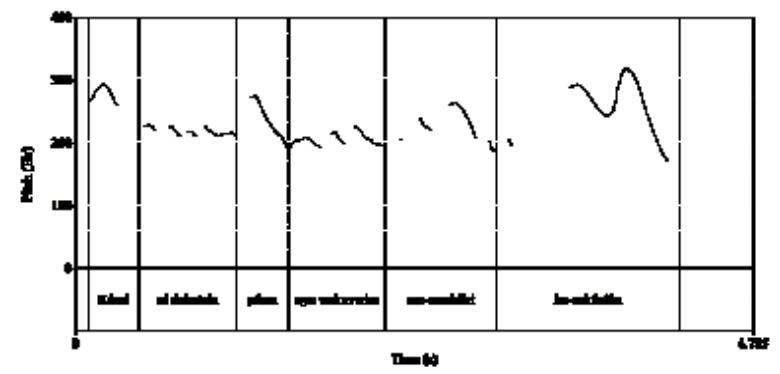

I will show that polar question formation cannot be accounted for with a rule inserting some intonational contour as it can in Chewa and Tumbuka. The phonological process is actually quite different. Rather than simply targeting the penultimate syllable for the shift in tone, the target of the prosodic manipulation in Kinyarwanda is connected to the location of underlying lexical tones within the utterance. My main claim is that Kinyarwanda targets the rightmost lexical high tone in the utterance as the edge of a prosodic domain. That edge is then marked by raising the high tone that is already there.

4.1. PATTERNS OF PROSODIC MANIPULATION. (16), (17), and (18) show the tonal patterns of three individual words that will be used in a sentence. Because of tone anticipation, the lexically low infinitive marker in (16) inherits the high tone from the verb stem, resulting in a HHL intonational pattern. The only other lexical high tone is the second syllable of (17). These words do not contain any long vowels, so there is no opportunity for a phonetic contour 
tone surfacing. Each word contains three moras, and thus there is a maximum of three tones that could surface in these words.

kú-rúm-a

INF-bite-ASP

'to bite'

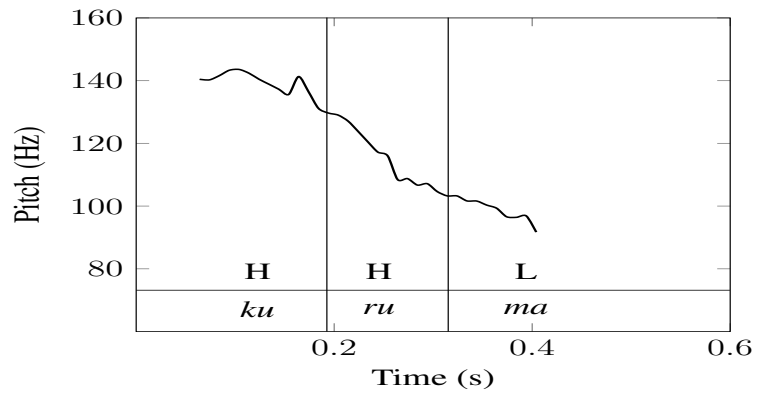

(17)

i-héne

9-goat

'goat'

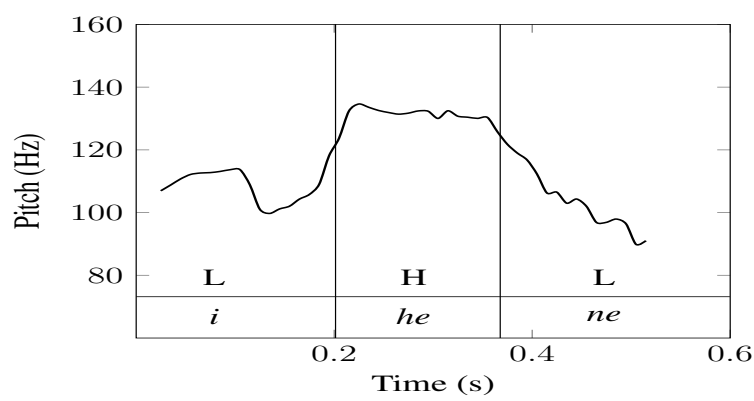

(18)
i-nyone
9-bird
'bird'

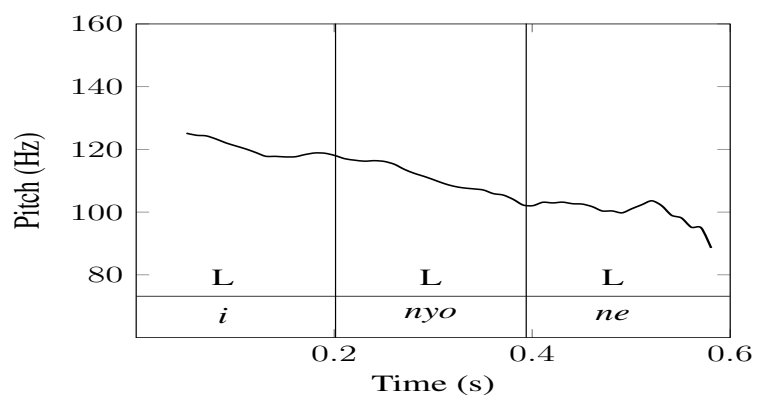

The lexical tones of these words in isolation differ from the tones that surface when they are used in an utterance. This supports the hypothesis that the right edges of prosodic words in Kinyarwanda are marked with a high tone while the right edge of the utterance is marked by deleting any high tones that may have existed. This is outlined in Section 2.3.

i-nyoné i-rum-yé i-héne

9-bird 3SG-bite-ASP 9-goat

'The bird bites the goat.'

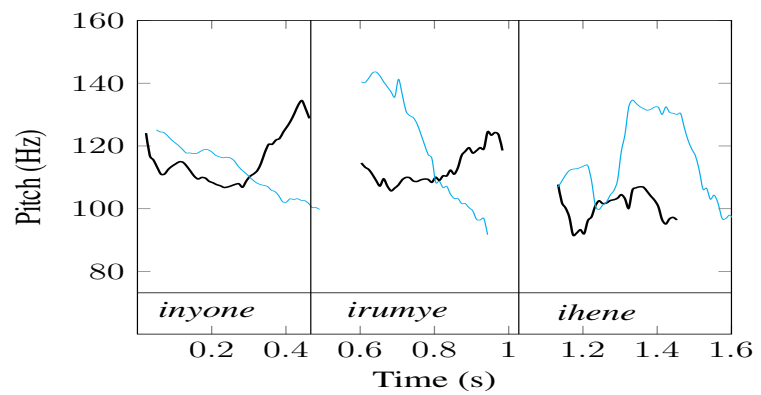


Each successive high tone in (19) is downstepped resulting in the lowering of high tones as the sentence progresses. In the question counterpart in (20), the word final high tones do not appear, and the final word of the sentence has an extra high (or raised high) tone.

i-nyone i-rum-ye i-hëne

9-bird 3SG-bite-ASP 9-goat

'Does the bird bite the goat?'

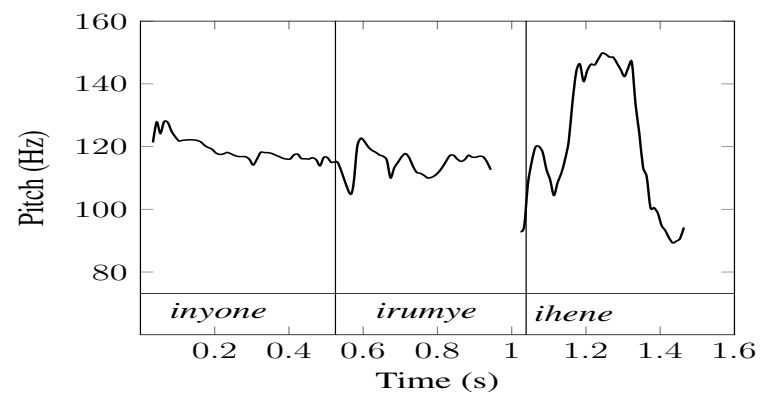

I will claim that the reason for the suppression of the word-final high tones in (19) and the raising of the lexical high tone in (20) is due to the creation of a prosodic domain ranging from $\mathrm{C}$ to the rightmost lexical high tone in the utterance. The high tone on the right edge of this domain also undergoes suspension of downstep.

A key reason that a local phonological process targeting the penultimate syllable of the utterance is inadequate to account for polar question formation comes from the fact that if we minimally change (19) so that the nominal with the high tone is in the subject position rather than the object position, the intonational contour of the question no longer resembles a rule that targets the penultimate syllable.

i-héné i-rum.yé i-nyoni

9-goat 3SG-bite-ASP 9-bird

'The goat bites the bird.'

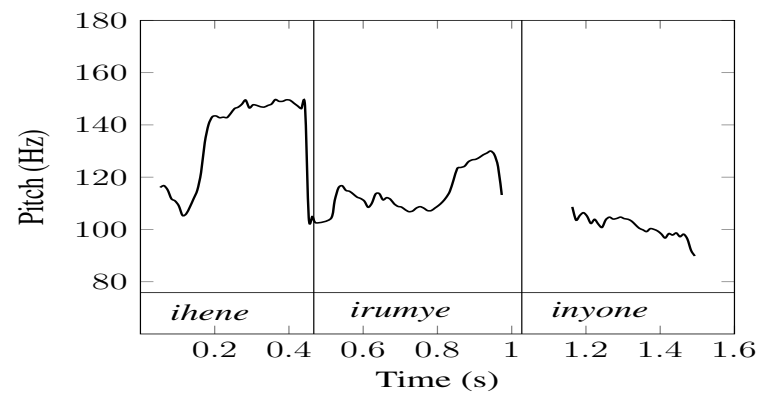


i-hëné i-rum-ye i-nyoni

9-goat 3sG-bite-AsP 9-bird

'Does the goat bite the bird?'

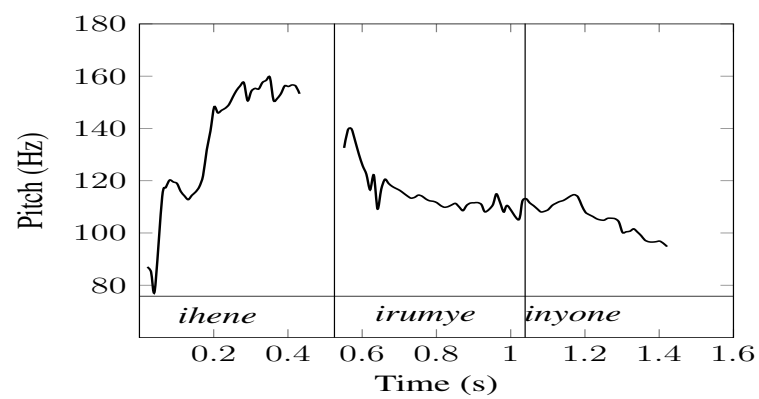

Because in these sentences the arguments only have one surface level high tone, we can observe here that high tone is raised in a question, regardless of where it occurs in the sentence. It is important to note that the verb in (19) and (21) also has a high tone that is not raised because it is a phrasal tone and not a realization for lexical tone. When there are multiple lexical high tones in the sentence, downstep is suspended and the rightmost high tone is raised to a higher pitch than lexical tone.

Similar to the situation described in Jita, when there are no lexical high tones in the utterance the penultimate syllable is targeted for the high tone. (13) is repeated below as (23).

a-rimó a-ra-jend-á kwi-soko

3SG-PROG 3SG-PROG-go-ASP LOC-market

'S/he is going to the market.'

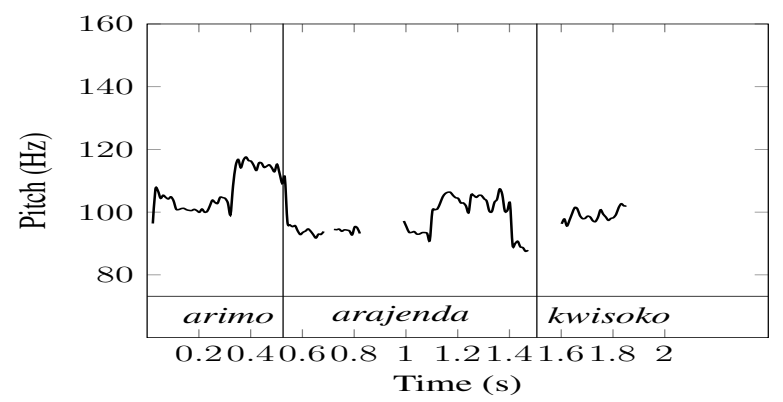

In this example, the only high tones that surface are those that mark the right edge of prosodic words. Individually, the verb and the nominal in object position do not have any high tones. The auxiliary arimo is difficult to categorize as it is mostly a function word and does not contribute lexical content. I am going to assume it doesn't have lexical tone, but gets the high tone observed here from a tonological operation having to do with tense.
ku-genda
INF-go
'to go'

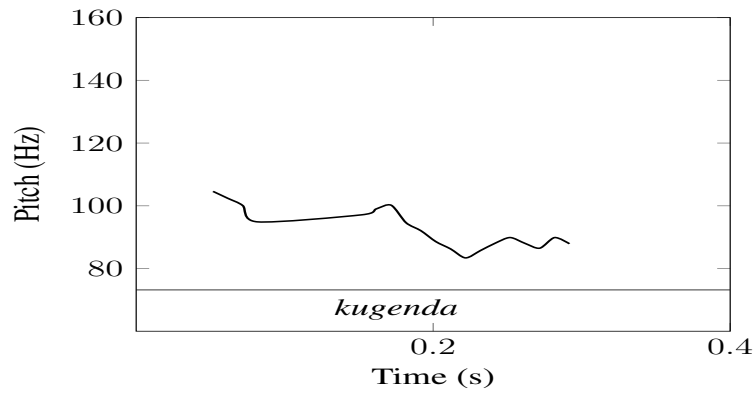




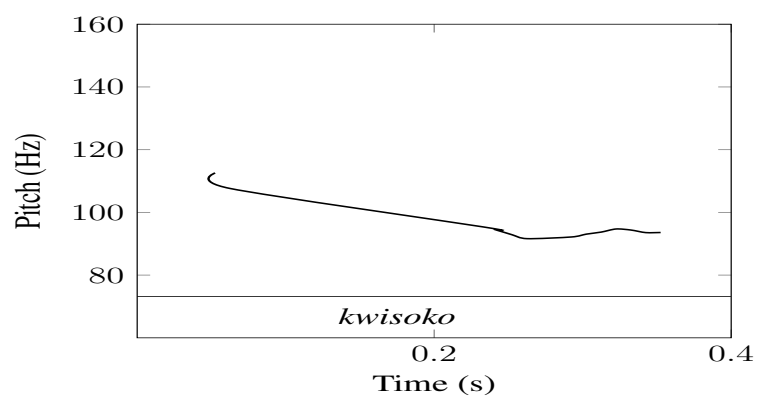

When (23) is made into a question, a high tone emerges in the penultimate syllable even though there is no lexical high tone in that location.

$$
\begin{array}{lll}
\text { a-rimó } & \text { a-ra-jend-a } & \text { kwi-sőko } \\
\text { 3SG-PROG } & \text { 3SG-PROG-go-ASP LOC-market }
\end{array}
$$

'is s/he going to the market?'

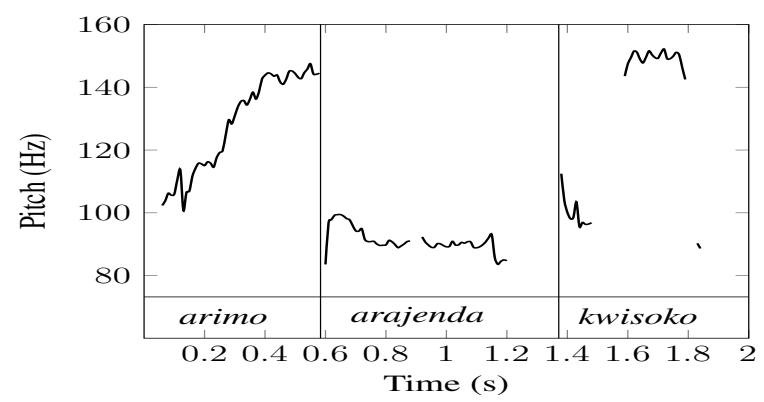

This polar question looks prosodically very similar to the others where lexical high tones exist. The high tone marking the right edge of the prosodic word on the verb is deleted and an extra high tone surfaces on the penultimate syllable. The finding that the penultimate syllable is the target of the high tone where no lexical high tones are present is not surprising. First, high tones in the utterance final position are not attested in Kinyarwanda, so the penultimate syllable is the rightmost possible target for the high tone. Second, this process is very similar to what is observed in Jita by Downing (1989). Recall that Jita polar questions with no lexical high tones raise the pitch of all syllables through the penult. Kinyarwanda seems to form polar questions in an analogous way to Jita. Where Jita raises the pitch of all syllables through the rightmost lexical high, Kinyarwanda raises only the rightmost lexical high. When no lexical high tones are present, Jita raises all syllables through the penult and Kinyarwanda raises only the penult.

4.2. TheORETIC ACCOUNT. The question remains as to why Kinyarwanda creates a prosodic domain between the complementizer and rightmost lexical high tone $(\mathrm{C}$ marking the left edge of the prosodic domain and the rightmost lexical high marking the right edge). To account for this, I will adopt a version of Richards (2010)'s analysis of wh-question prosody. This will require deviating from polar question in Kinyarwanda to a brief explanation of wh-question formation.

A central claim of Richards (2010) is that all languages are trying to create a prosodic structure for wh-questions in which the wh-phrase and the corresponding complementizer are separated by as few prosodic boundaries as possible: 


$$
\left[{ }_{\phi} \mathrm{C}\right]\left[[ _ { \phi } ] \left[\left[_{\phi}\right]\left[\left[_{\phi} w h\right] \rightarrow{ }_{\phi} \mathrm{C} \quad \text { wh }\right]\right.\right.
$$

Languages can either accomplish this by combining multiple phonological phrases to create one prosodic domain that contains both $\mathrm{C}$ and the wh-word (wh-in-situ), or with overt movement. For instance, Chichewa has initial complementizers and Minor Phrase boundaries to the right of certain XPs. Kinyarwanda is like Chichewa in that is has the possibility for wh-insitu. Following Richards (2010), the ability for wh-in-situ should mean that the language has both initial complementizers and marks the right edges of XPs as prosodic boundaries. I have already shown in Section 2.3 that Kinyarwanda marks the right edges of words as prosodic words, and we see that Kinyarwanda also has initial complementizers:

nda-nézewe ko a-ta-kuvúga

1SG-be.happy C 3SG-NEG-talk

'I'm happy that he doesn't talk.'

The complementizer $k o$ that introduces the embedded clause appears at the left edge of that clause. This combination of initial $\mathrm{C}$, prosodic boundaries at right edges, and wh-in-situ means that Kinyarwanda should also create some prosodic domain that includes both $\mathrm{C}$ and the whword. This is indeed the case, and the the prosodic domain that Kinyarwanda forms between $\mathrm{C}$ and the wh-word resembles a prosodic pattern found in Japanese.

a-rimó a-ra-jend-á kwi-soko

3SG-PROG 3SG-PROG-go-ASP LOC-market

'S/he is going to the market.'

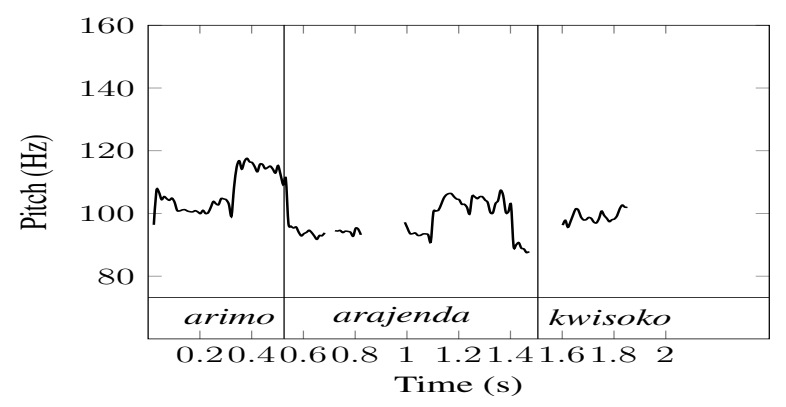

(30) a-rimó a-ra-jend-a he-he

3SG-PROG 3SG-PROG-go-ASP where-RED

'Where is s/he going?'

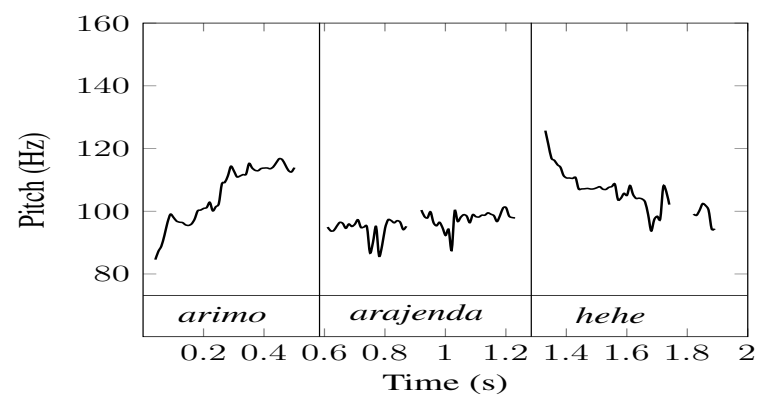

The prosodic patterns of wh-questions in Kinyarwanda mirror those of polar questions, except for the fact that polar questions lack an overt wh-word. Rather than a wh-word that serves as the target for the high tone, polar questions must rely on some other part of the utterance. 
Because Kinyarwanda marks the right edge of prosodic words with a high tone, the simple transitive sentence in (19) can be parsed into the following prosodic phrases: ${ }^{1}$

$$
\begin{aligned}
& \text { [ }{ }_{\phi} \text { i-nyoné ] [ }{ }_{\phi} \text { i-rum-yé ] [ }{ }_{\phi} \text { i-héne ] } \\
& \text { 9-bird 3SG-bite-ASP 9-goat }
\end{aligned}
$$

Here the right edge of each prosodic word is marked with a boundary tone, but the utterance final boundary tone is deleted as was noted in Section 2.3.

In a polar question, rather than targeting a wh-word with a high tone, instead the target becomes the rightmost lexical high tone. Because Kinyarwanda does not have rightward tone spreading, the next best option for creating the largest prosodic domain possible between $\mathrm{C}$ and the right edge of the utterance is the rightmost lexical high tone that exists in the sentence. We see this in the polar question counterpart:
a. $\quad{ }_{\phi} \mathrm{C}$ i-nyoné $]\left[{ }_{\phi}\right.$ i-rum-yé $]\left[{ }_{\phi}\right.$ i-héne ] 9-bird 3SG-bite-ASP 9-goat
'Does the bird bite the goat?'
b. [ ${ }_{\phi} \mathrm{C}$ i-nyone i-rum-ye i-hëne ]

In (32a), each prosodic word is grouped in its own prosodic domain with a boundary tone marking the right edge (this is the declarative). To form the polar question in (32b), the domain between $\mathrm{C}$ and the rightmost lexical high is grouped into a single prosodic phrase. This means that there are no longer prosodic boundaries between the individual words and thus there are no boundary tones.

The pitch track in (32) shows that the observed tonological pattern fits this analysis. All boundary tones are suppressed and the rightmost lexical high tone is raised. The prosodic domain established in the polar question construction is highlighted.

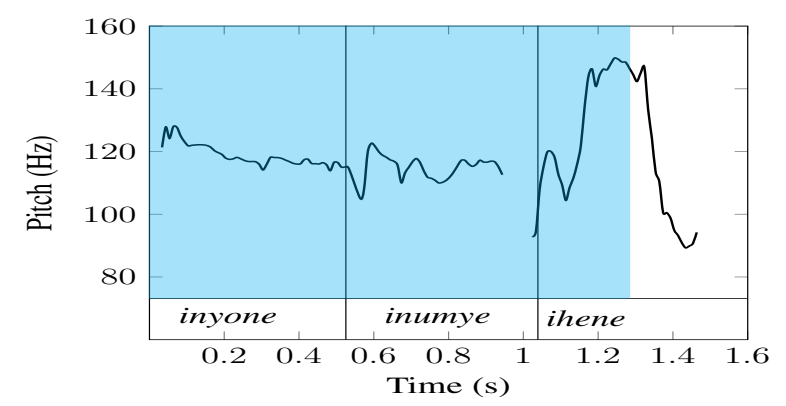

Figure 1. Prosodic domain of Kinyarwanda polar questions

4.3. FURTHER DISCUSSION AND IMPLICATIONS. One possible explanation for the suppression of lexical high tones that serve as the right edge of the prosodic domain of polar questions has to do with contrast. Because the manipulation of tone is the only way to distinguish declaratives and polar questions in Kinyarwanda, speakers may accentuate those tonal manipu-

\footnotetext{
${ }^{1}$ For Richards the Minor Phrase is the lowest level of phonological phrasing that can occur. It is not especially important for my analysis what these prosodic phrases are labeled as, so I am calling them prosodic words as they correspond with syntactic word boundaries.
} 
lations in order to ensure polar questions are interpreted as separate from declaratives.

Another possibility for avoiding raising the verb-final tone in polar questions is to disambiguate any tonological functions within the prosodic domain that contains the raised high tone. Tone in Kinyarwanda serves lexical, grammatical, and prosodic roles. In addition to this, tones often spread/shift, and are added and deleted. Because verbs in Kinyarwanda already bear such a great tonological burden, it may increase the functional load of the verb too much to add another tonological process that isn't reliant on an already-present tone.

5. Conclusion. In this paper I have provided a detailed descriptive generalization of polar question formation in Kinyarwanda and shown that the rightmost lexical high tone is raised for utterances with at least one lexical high tone, and the penultimate syllable is raised for utterances with no lexical high tones. This places Kinyarwanda within a greater typological system of polar question formation in Bantu languages. I have shown that polar questions in Kinyarwanda pattern in a very similar to way to Jita and that the differing tonological operations available to these languages can account to the observable differences in polar question formation.

An interesting finding of this work is that Kinyarwanda forms a prosodic domain with a syntactic position as one edge and a phonological feature as the other edge. The complementizer is certainly a syntactic position, but the rightmost lexical high tone is not. Having the rightmost lexical high tone serve as a boundary of the prosodic domain suggests that speakers want the biggest possible domain, but must rely on an already existing tone to mark its edge

More work needs to be done with regards to whether there is a minimum or maximum size to this prosodic domain. I have found that polar question formation in Kinyarwanda is a much more complicated process than a simple phonological rule stipulating a floating or spreading tone, or a rise-fall contour at the end of the utterance. It may be the case that polar question formation in other Bantu languages are also more complicated than meets the eye, and could benefit from further analysis of how tonological operations apply with certain syntactic boundaries.

\section{References}

Connell, Bruce. 2016. Tone and intonation in Mambila. In Laura J. Downing \& Annie Rialland (eds.), Intonation in African tone languages. 131-166. Berlin: Mouton de Gruyter.

Downing, Laura J. 1989. Tone in Jita questions. In Eyamba G. Bokamba (ed.), The contribution of African linguistics to linguistic theory: Proceedings of the 20th Annual Conference on African Linguistics (Studies in the Linguistic Sciences, volume 19.2). 91-114. UrbanaChampaign: University of Illinois. http://hdl.handle.net/2142/9370.

Downing, Laura J. \& Al Mtenje. 2011. Un-Wrap-ing prosodic phrasing in Chichewa. Lingua 121(13). 1965-1986. https://doi.org/10.1016/j.lingua.2010.12.003.

Goldsmith, John A. \& Fidèle Mpiranya. 2010. Rhythm, quantity, and tone in the Kinyarwanda verb. In John A. Goldsmith, Elizabeth Hume \& Leo Wetzels (eds.), Tones and features: Phonetic and phonological perspectives. 25-49. Berlin: Mouton de Gruyter.

Hyman, Larry M. 1990. Boundary tonology and the prosodic hierarchy. In Sharon Inkelas \& Draga Zec (eds.), The phonology-syntax connection. 109-125. Chicago: University of Chicago Press.

Hyman, Larry M. 1993. Register tones and tonal geometry. In Keith Snider \& Harry van der 
Hulst (eds.), The phonology of tone: The representation of tonal register. 75-108. Berlin: Mouton de Gruyter.

Kanerva, Jonni M. 1990. Focusing on phonological phrases in Chichewa. In Sharon Inkelas \& Draga Zec (eds.), The phonology-syntax connection. 145-161. Chicago: University of Chicago Press.

Kimenyi, Alexandre. 1980. A relational grammar of Kinyarwanda. Berkeley: University of California Press.

Kimenyi, Alexandre. 2002. A tonal grammar of Kinyarwanda: An autosegmental and metrical analysis (Studies in Linguistics and Semiotics 9). Lewiston, NY: Edwin Mellen Press.

Maddieson, Ian. 2005. Tone. In Martin Haspelmath, Matthew S. Dryer, David Gil \& Bernard Comrie (eds.), World atlas of language structures. 58-61 Oxford: Oxford University Press.

Myers, Scott. 1996. Boundary tones and the phonetic implementation of tone in Chichewa. Studies in African Linguistics 25(1). 29-60.

Myers, Scott. 2003. F0 timing in Kinyarwanda. Phonetica 60(2). 71-97. https://doi.org/10.1159/000071448.

Odden, David \& Lee Bickmore. 2014. Melodic tone in Bantu: Overview. Africana Linguistica 20. 3-14.

Rialland, Annie. 2007. Question prosody: an African perspective. In Tomas Riad \& Carlos Gussenhoven (eds.), Typological studies in word and sentence prosody. 35-62. Berlin: Mouton de Gruyter. https://doi.org/10.1515/9783110207569.35.

Richards, Norvin. 2010. Uttering trees. Cambridge, MA: MIT Press.

Selkirk, Elisabeth. 1980a. Prosodic domains in phonology: Sanskrit revisited. In Mark Aronoff \& Marie-Louise Kean (eds.), Juncture. 107-129. Saratoga, CA: Amma Libri.

Selkirk, Elisabeth O. 1980b. The role of prosodic categories in English word stress. Linguistic Inquiry 11(3). 563-605. https://www.jstor.org/stable/4178179. 